\title{
Single and dual incision technique for acute distal biceps rupture: clinical and functional outcomes
}

\author{
Claudia Guglielmino ${ }^{1,2}$ \\ Paolo Massimino ${ }^{2}$ \\ Francesco Ioppolo 2 \\ Sergio Castorina ${ }^{1,2}$ \\ Giuseppe Musumeci ${ }^{1}$ \\ Angelo Di Giunta ${ }^{2}$
}

1 Department of Biomedical and Biotechnological Sciences, Human Anatomy and Histology Section, School of Medicine, University of Catania, Catania, Italy

2 Polyclinic "G.B. Morgagni” Mediterranean Foundation, Orthopedics Traumatology and Rehabilitation Unit, Catania, Italy

\section{Corresponding author:}

Giuseppe Musumeci

Department of Biomedical and Biotechnological

Sciences, Human Anatomy and Histology Section,

School of Medicine, University of Catania

Via S. Sofia 87

95125 Catania, Italy

E-mail: g.musumeci@unict.it

\section{Summary}

Background: Distal bicep tendon injuries are a traumatic event though rather rare. The pathogenesis is not entirely clear. The most common cause for injury is an unexpected load on the biceps when the elbow is in an extended position. Although several studies have provided insight into the pathogenetic processes of the lesion, the literature suggests to treat all injuries surgically (whether partial or total) if there is high functional demand.

Methods: Between January 2006 and March 2016 were studied 20 patients surgically treated for a disconnected distal bicep, 15 with a total lesion and 5 with a partial lesion. The patients were divided into 2 groups. Surgical access with single incision was performed on 13 patients while a double surgical access was performed on 7 patients. The clinical and functional results were studied using an Ewald System Score (ESS).

Results: In both groups, the most rapid improvement was achieved for the parameters of pain and deformity with excellent results, while those of function and movement were normalized as gradual and progressive over next 2 months.

Conclusion: The clinical and functional outcomes during the follow-up examination after surgery showed excellent results in patients treated with both types of surgical procedures.

KEY WORDS: distal biceps, endobutton, ESS, single incision, surgical procedure.

\section{Introduction}

Distal bicep tendon injuries are a traumatic event though rather rare. They account for about $4 \%$ of all bicep brachial injuries that occur as a result of an acute or degenerative trauma. The distal insertion site of the tuberosity radius is most frequently involved during the break. Most patients are males from 30 to 60 years old, comprising $95 \%$ of the cases $^{1} ; 86 \%$ of the cases involves the dominant arm of the patient. Smokers are seven and a half times more likely to suffer from this injury than non-smokers. The vasoconstrictor and deoxygenating effects of nicotine degenerate the tendon, which increases the likely hood of injury. The pathogenesis of distal head bicep ruptures is not entirely clear. The most common cause for injury is an unexpected load on the biceps when the elbow is in an extended position. This situation creates a complete avulsion in the tendon at the distal insertion site of the tuberosity radius. This type of injury affects top athletes either as a chronic or acute disease. In chronic cases (tendinosis), the lesion is due to an unfavourable balance between collagen synthesis stimulated by the physical activity and the effect of wear on the tendon structures (structural catabolism of proteins, tissue, repeated friction against surrounding structures the tendon, trauma from sudden and intense tractions) due to repeated stress. In addition, degeneration due to ageing involves modification of the tendons' cellular matrix due to both qualitative and quantitative alterations. The hypovascularity phenomena due to a reduction in blood supply in the arteries and arterioles results in a reduction of trophic exchanges with hypoxic phenomena. Calcium and/or lipid deposits are frequently observed with the progression of age. In addition to unchangeable intrinsic factors, acute lesions in the bicep tendon may be related to a number of factors such as: intense workouts, insufficient re- 
covery time, inadequate stretching, use of inadequate equipment. Anatomical factors, such as dysmetria, malalignment, abnormal conformations and bony prominences, can increase mechanical stress favoring the onset of injury.

Other important comorbidities in the pathogenesis of these lesions results from metabolic factors such as hypercholesterolemia, diabetes mellitus, thyroid disease, obesity and metabolic syndrome increase the risk of tendon degeneration. Thyroid hormones are involved in the metabolism of collagen, which is important for tendons growth ${ }^{2}$. Diabetes is associated with a higher frequency of tendon calcifications. Obesity can reduce or undo the beneficial effects of moderate exercise on the tendon. In fact, injuries occur 6.6 times more in obese patients ${ }^{3}$. Hyperlipidemia promotes the deposit of xanthomas in the tendon that leads to tendon degeneration, thus increasing likelihood of injury. A crucial component in metabolic tendon injuries is the prolonged silent symptom phase, where anatomical damage is present and progressively evolving ${ }^{4}$. In addition, drug exposure appears to contribute to an increased risk of tendon rupture. Quinolones, as well as corticosteroids, appear to inhibit the production of collagen increasing the chance of tendon rupture ${ }^{5,6}$.

Although several studies have provided insight into the pathogenetic processes at the base of the lesion, and clarified the role of risk factors, the literature suggests to treat all injuries surgically (whether partial or total) if there is high functional demand ${ }^{7}$. A conservative treatment results in a $40-50 \%$ reduction in elbow flexion strength and forearm supination for complete ruptures of the distal bicep tendon. Most lesions affect almost exclusively males, while partial lesions have been reported in both sexes equally. Partial lesions normally occur in patients around 50 years of age, while complete injuries affect patients around 40 years of age. For both types of lesions, the dominant limb is involved in the majority of cases. As mentioned above, distal bicep injuries are common in athletes who play sports, especially contact or sports involving throwing, including American football, rugby and tennis. A special situation is seen in many body builders, where at the base of the tendon injury there is an alteration in the constitution of the tendon structure, because of the use of anabolic steroids ${ }^{8}$, which is a degenerative process, added to the increased muscle mass, sometimes disproportionate, which increases the traction forces in the tendon. Lesions are generated as a result of an eccentric contraction in the bicep while lifting heavy loads, whereby, the patient clearly describes the traumatic moment as a sudden feeling of crackling-lash in the front face of the elbow followed by a strong localized pain. The symptoms, understood by considering the patient history and through semiotics, are seen in the presence of an empty area with an empty feeling under finger pressure during elbow flexion and forearm supination, called negative sign Hook, and pain while performing these movements against resistance. The presence of a partial lesion, which is less common than a total lesion, can involve the insertion site in the radial tuberosity and in the myotendinous junction. For these reasons it is more difficult to diagnose a partial lesion, though imaging techniques (MRI, Ultrasound, $\mathrm{RX}$ in selected cases) may help to clarify the suspected diagnosis. Both types of lesions should be treated surgically by restoring tendon continuity for full restitutio ad integrum. The endoscopic technique is an important tool that is becoming increasingly popular in the area of diagnostics and therapeutic treatment, especially in treating a partial tear. This technique allows us to dynamically evaluate the distal insertion of the bicep tendon, and provides a clear picture of the disease through a minimally invasive procedure.

It is important also to quantify the percentage of the involved tendon. Data from the literature confirms that injuries involving less than $50 \%$ tendon insertion do not require surgical treatment, because they do not constitute functional deficits ${ }^{9,10}$. Whereas, tendon ruptures involving more than $50 \%$ of tendon insertion site require reinsertion using the techniques previously described. Classifying the lesion by the endoscopic technique can help the surgeon in managing this injury. Grade "0" lesions show up as a tendinosis of the distal bicep with pain at the supination and flexion under resistance. In grade "1" lesions, we report a partially torn tendon with pain and reduction of strength in movements of supination and flexion against resistance. In grade "2" lesions, there is a combination of avulsion in the short and long biceps' stringy tendon, and minimal muscular retraction. Therefore, a grade " 2 " lesion is associated with pronounced reduction of strength in flexion and supination against resistance and yields a positive Hook test. In grade " 3 " lesions, there is a complete rupture of the tendon involving the fibrous tendon and leading to muscle retraction. In addition, there is intense weakness in supination and flexion, positive Hook test and the cubital fossa appears "empty." In Grade "4" lesions, there is a delay in diagnosing the tendon rupture. There remains weakness at the movement of supination and flexion against resistance, Hook positive test and proximal retraction of the muscle belly. It is possible to differentiate treatment based on the classification of the lesion.

In grade " 0 " lesions the treatment is no operative, grade "1" and "2" lesions need surgical treatment, and grade " 3 " lesions need surgical repair of the fibrous tendon. Grade "4" lesions require autograft or allograft reconstruction, when the retraction/degeneration do not allow the combination of lesion stumps.

The purpose of this study is to retrospectively evaluate the clinical and functional results of surgical treatment with single and dual access for distal lesions of the brachial bicep.

\section{Materials and methods}

Between January 2006 and March 2016, at the Policlinico "G.B. Morgagni" of Catania, we studied 20 patients according to international ethically standards ${ }^{11}$, 


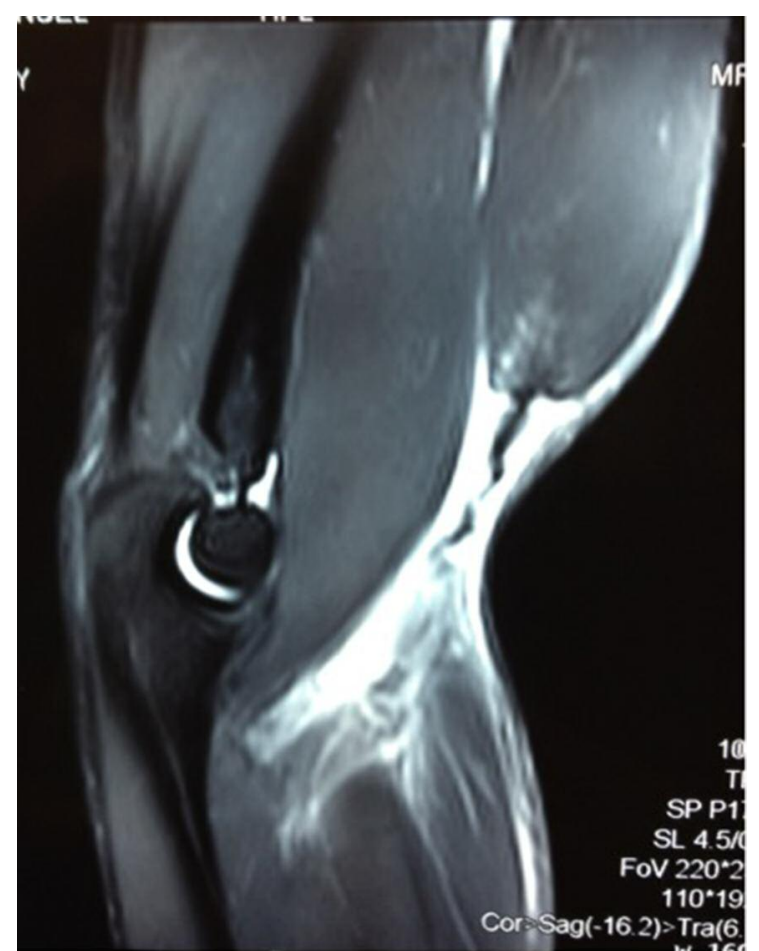

Figure 1. MRI vision of the avulsion of the biceps tendon from the radial tuberosity.

surgically treated for a disconnected distal biceps lesion, 15 with a total lesion and 5 with a partial lesion. All subjects were males and underwent a preoperative MRI (Fig. 1). The age range was between 24-53 years, with a mean age of 36.3 years. The affected side was the right in 14 cases $(70 \%)$, and the left in 6 cases $(30 \%)$. The injury was unilateral in 18 cases $(90 \%)$, while it was bilateral in 2 cases $(10 \%)$. The elapsed time between the traumatic injury and surgical treatment was between 2 and 30 days. In 3 cases $(14 \%)$ patients were suffering of hypercholesterolemia, while in 5 cases the patients were smokers (25\%). The patients were divided into 2 groups. In group 1 (10 cases), were included subjects that experienced surgical incision on the volar side of the radial tuberosity. In group 2 (10 cases), patients underwent double surgical incision one on the volar side of the radial tuberosity and the other to the distal third of the arm.

\section{Surgical treatments}

Different types of surgical treatments (Figs. 2-5), as previously described ${ }^{12}$, including the suture tendon in 6 cases $(30 \%)$ were performed, these included use of the cortical radial suspension systems with fixed length (Endobutton) in 7 cases (35\%), use of metallic anchors on the radial tuberosity in 4 cases $(20 \%)$ and use of a cortical radial suspension system with editable loop length (ZipLoop, ToggleLoc) in 3 cases (15\%). Surgical access with single incision at the level of the radial tuberosity was performed on 13 patients $(65 \%)$, while a double surgical access, both to the radial tuberosity and to the distal third of the arm was performed on 7 patients (35\%). All the patients underwent an X-ray (Fig. 6) to the forearm, anteroposterior and latero-lateral projection post operatively. Subsequently, the affected arm was immobilized with a tutor type Gilchrist, valve pinstripe at the brachial forearm or elbow brace locking the flexion at $90^{\circ}$ and maintained for 3-4 weeks. Following this pe-

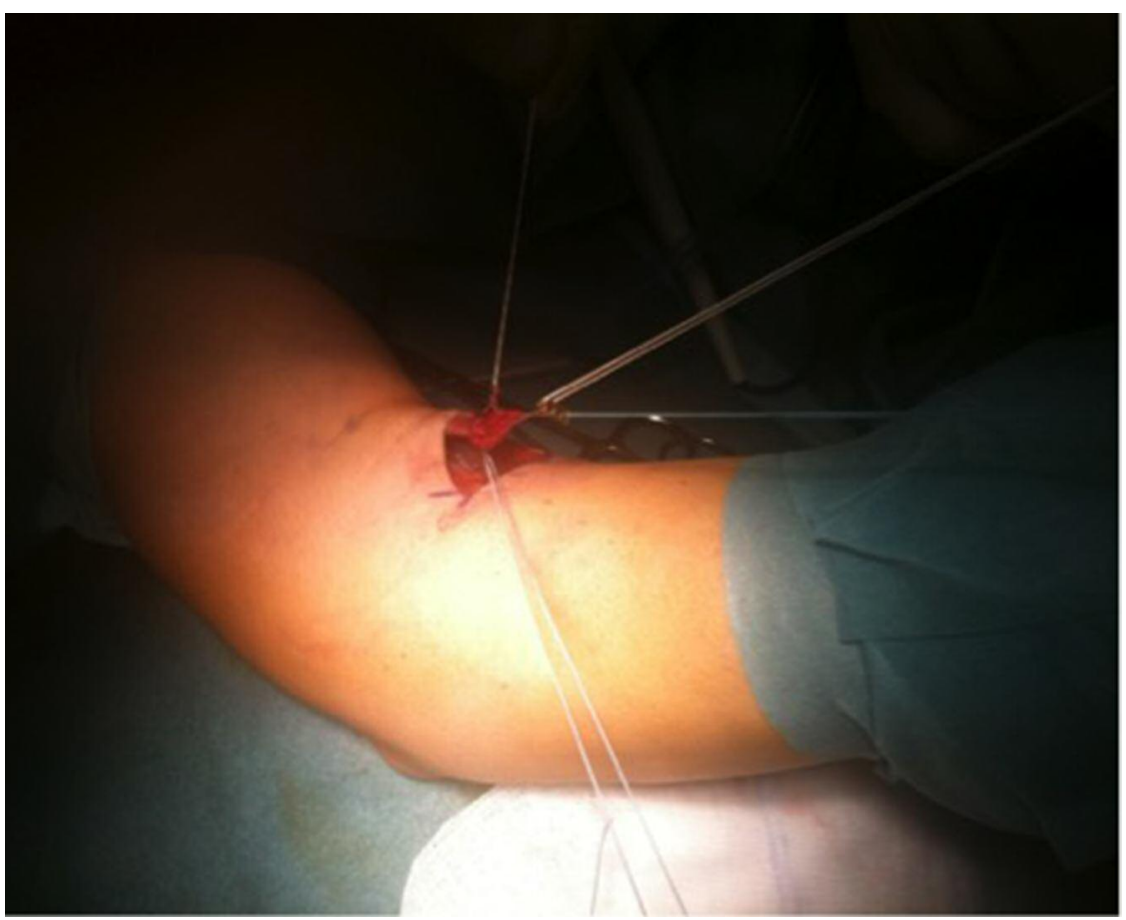

Figure 2. The tendon is fixed with two sutures of different colour with a Krackow suture and at the end secured with an Endobutton free loop (single incision technique). 


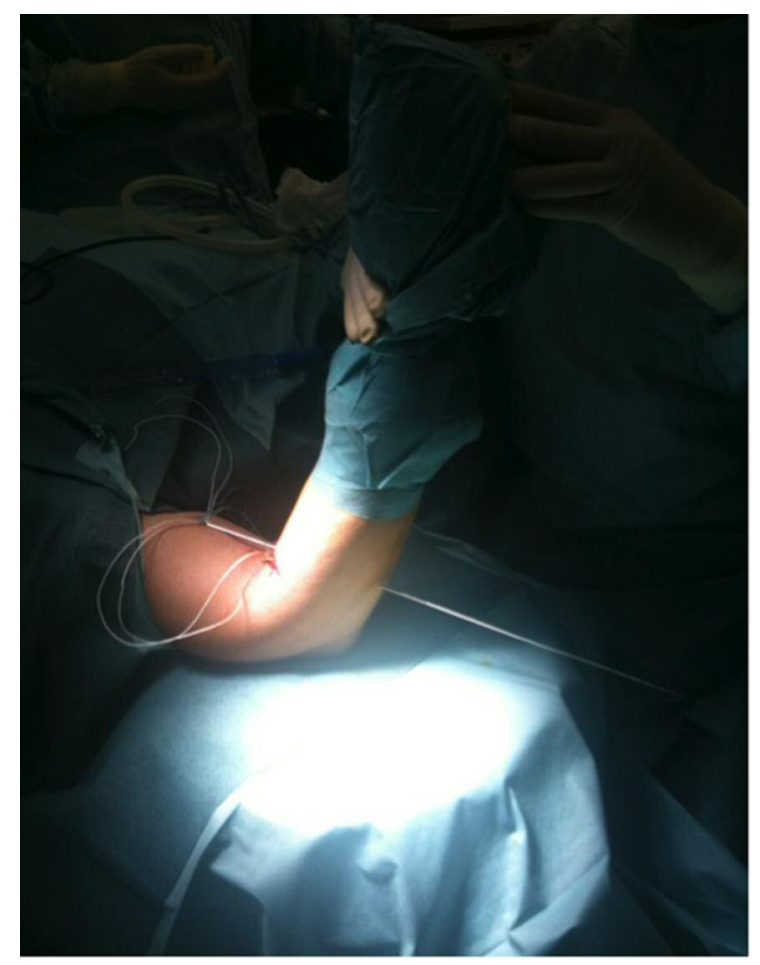

Figure 3. With a $\mathrm{K}$ wire the tendon armed with the suture and the endobutton is passed with the control of fluoroscopy true the radial tuberosity (single incision technique).

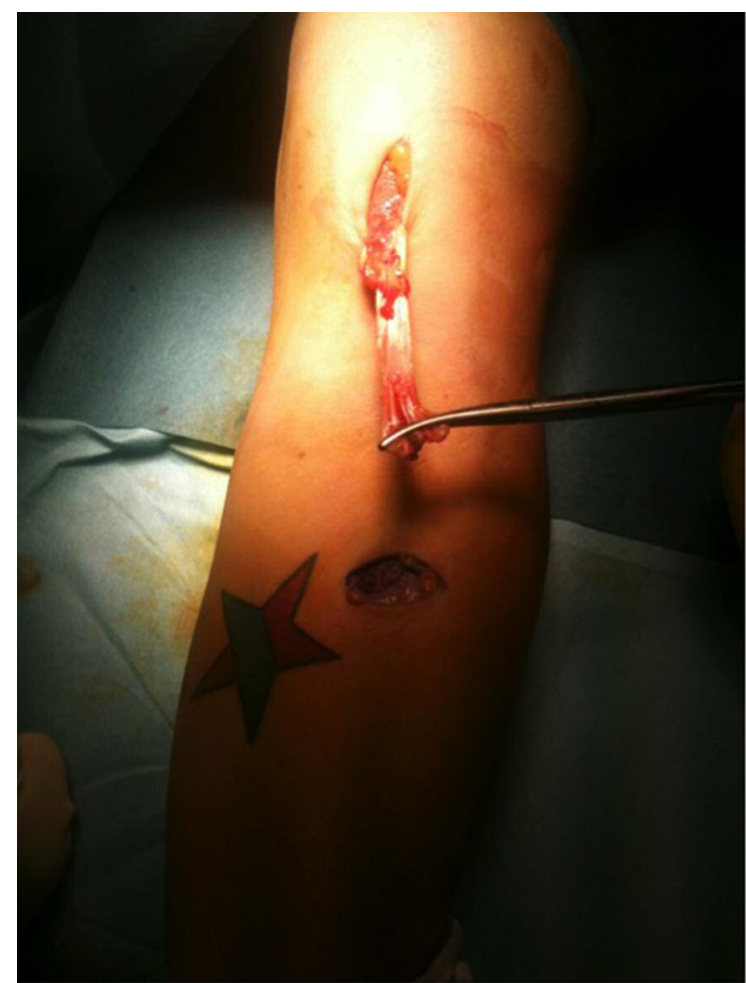

Figure 4. Release of the bicep tendon with the double incision technique.

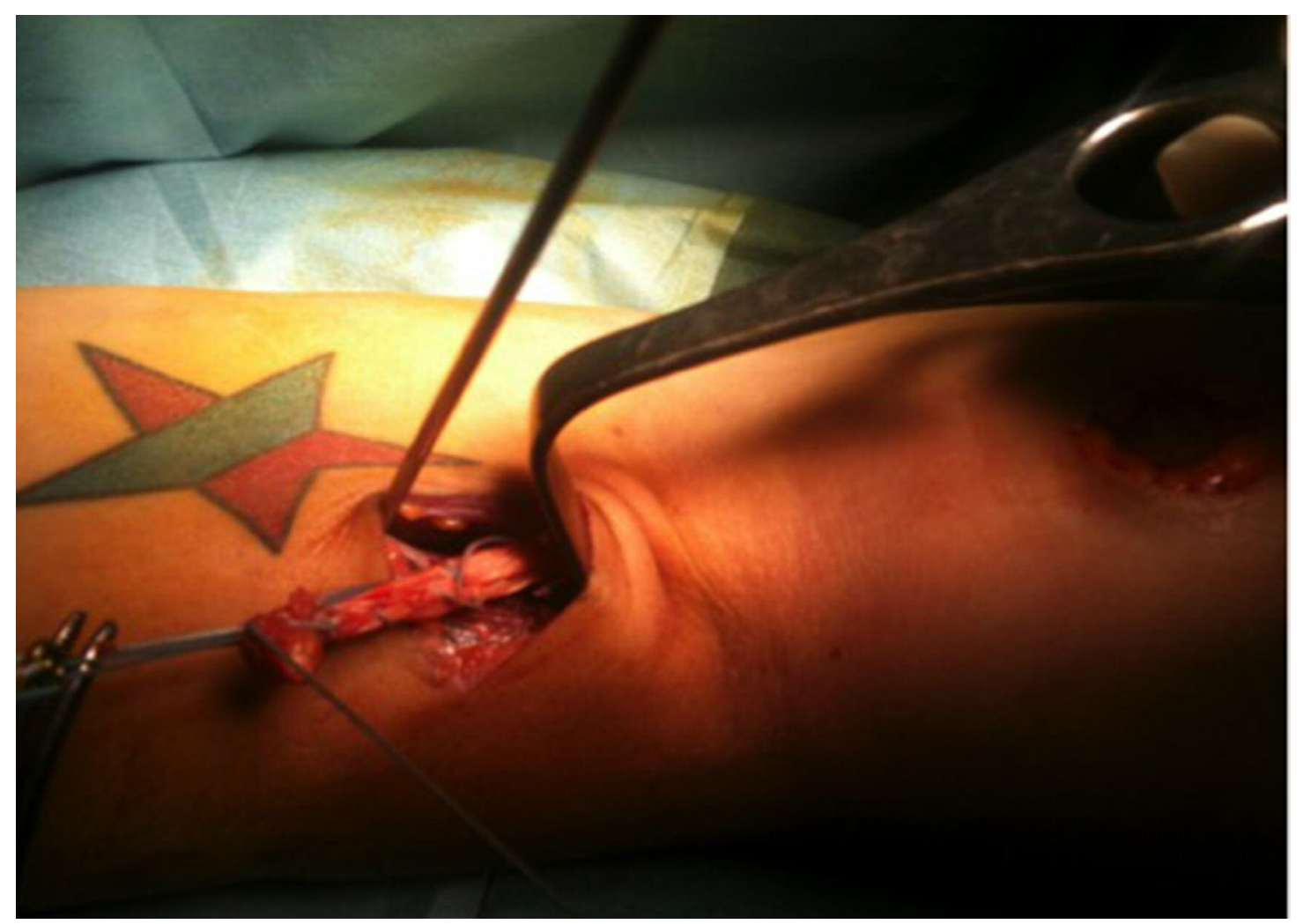

Figure 5. The bicep tendon is passed true the forearm incision and armed with the 2 sutures. 


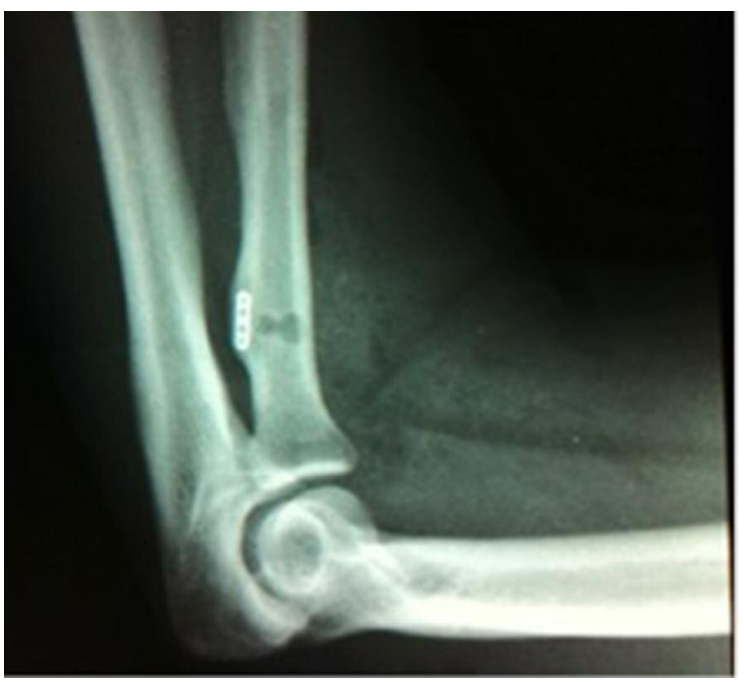

Figure 6. X-ray to the forearm, anteroposterior and laterolateral projection post operatively.

riod, the patient begins a rehabilitation program after the first month of follow up, than he will have the second follow up after 3 months, and can return to full sports and work about 6 months after surgery to return to sports. The clinical and functional results were studied using an Ewald System Score (ESS), which included an evaluation score of 100 points covered by: pain, function, movement and deformity, with a follow-up clinical and functional treatment performed 6 - 12 - 24 months after surgery without any observed complication.

\section{Statistical analysis}

Statistical analysis was performed using SPSS software (SPSS $®$ release 16.0, Chicago, IL, USA) as previously described ${ }^{13}$. Data were tested for normality with the Kolmogorov-Smirnov test. All variables were normally distributed. Results comparisons between two means were tested with t-student test. p-values of less than 0.05 were considered statistically significant; $p$-values of less than 0.01 were considered statistically highly significant. Data are presented as the mean \pm SD. Data were analyzed by three blinded investigators.

\section{Results}

The results of the ESS questionnaire for group 1 patients treated for insertion of the bicep injury were: "Excellent" in 8 patients, "Good" in 2 patients, and no cases with "Fair" and "Poor" results $(p<0.05)$.

Whereas, in group 2 patients the results were: "Excellent" in 8 patients, "Good" in 2 patients, and no cases with "Fair" and "Poor" results $(p<0.05)$. These data confirm that there are not significant differences between the two groups.

In both groups, the most rapid improvement was achieved for the parameters of pain and deformity with excellent results, while those of function and movement were normalized as gradual and progressive over next 2 months. Only one patient developed acute complications, in fact the patient reported a stupor sensorimotor of the interosseous nerve that persisted for 10 months. This patient was treated with dual access technique (group 2). This data is not statistically significant. Patients evaluated in both groups during follow-up had all resumed their daily activities and work 2 months after surgery. Patients who practiced contact sports (e.g. American football) resumed full activity 6 months after treatment. For both groups of patients heavy lifting and perform vigorous activity was prohibited for 4-5 months.

\section{Discussion}

When the suspect is a tendon biceps tear we must make a differential diagnosis among the causes of pain in the anterior region of the elbow among bursitis, tendinitis, or forearm lateral cutaneous nerve syndrome. Imaging allows us to obtain useful diagnostic information. An X-ray of the elbow is performed to assess the presence or absence of an avulsion from the radial tuberosity and the presence of a tuberosity hypertrophy that may be indicative of chronic degenerative process.

$\mathrm{MRI}$ is still the gold standard for such injuries by giving detailed information about the lesion, tendon and muscle quality characteristics, dislocations and evidence of incomplete injury, even if the diagnosis is basically clinical. Treatment can be either conservative or surgical. Conservative treatment is usually reserved for older patients and patients with poor functional demand. Most patients require surgical treatment, because of the aesthetic appearance of a retracted muscle, the reduced strength and endurance. This procedure, as already described above, makes use of intramedullary fixation with transosseous sutures, and anchors and fixations with cortical button. Furthermore, it is appropriate to proceed with surgery two to three weeks after the trauma to obtain the best results, before the tendon retracts proximally.

Two main types of surgical accesses have been described. The Henry anterior approach with a single incision on the volar side of the elbow (e.g. medialproximal longitudinal, transverse, lateral-distal) at the height of the radial tuberosity. Over the years, a miniopen technique was also developed, which is characterized by a $2-3 \mathrm{~cm}$ transverse skin incision (Fig. 7) centred on the anterior cubital crease. The tendon stump is freed through the incision aided by squeezing the bicep muscle. The stump tendon, which is usually edematous, may be pulled out of the wound quite easily. If it does not emerge it can be pulled out with a finger. It is useful to place a suture in the tendon stump to control the tendon and prevent damage from the forceps. The tendon stump is regularized and properly prepared before replanting ${ }^{12}$. The dual accesses technique is realized in the volar region of the elbow, or possibly an access of about $5 \mathrm{~cm}$ in the 


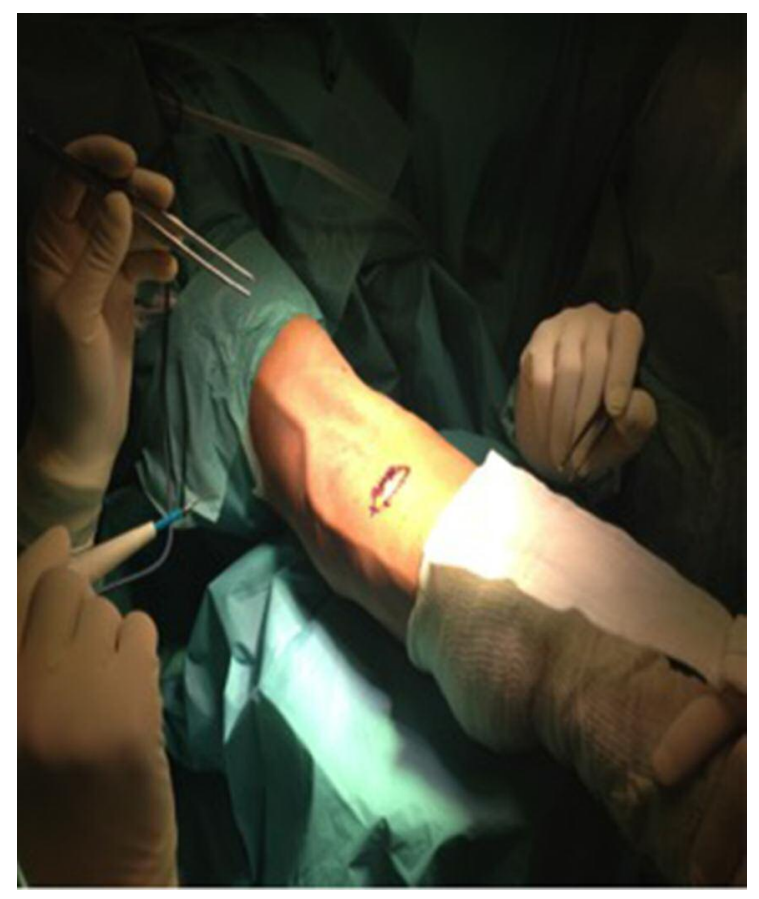

Figure 7. 2-3 cm transverse skin incision.

distal third on the ventral side of the arm is used ${ }^{14}$. The main advantage of the anterior approach with single access is the presence of a single incision that can be extended, if necessary, to improve access to the radial tuberosity. This single access surgical approach is technically more demanding than the dual accesses approach, and there is greater risk of damage to the posterior interosseous nerve. The dual access technique uses two small incisions to identify the distal end of the bicep tendon. The incision is performed on the volar side of the forearm through the muscle fibers placed above the radial tuberosity with the forearm held in full pronation. This technique with dual access can induce the formation of an ectopic bone between the proximal radius and ulna by accidentally exposing the radial side of the ulna. To avoid this complication, which in some patients has caused synostosis, it is important to maintain the muscle dissection plane adjacent to the radial passing the tendon around the forearm rear face to prevent the accidental injury of the anconeus muscle from the radial side of the ulna ${ }^{15}$. In the fixing suspension system treatment, the tendon tear is identified and the proximal retracted tendon is fixed with fiberwire and tigerwire with a Krackow suture and put back with the inout technique at the radial tuberosity with an endobutton free loop or securing it with the Zip Loop system. In treatment with fixation anchors, the retracted tendon is attaches to the tuberosity of the radius with the still armed 2 fiberwire wires. Regarding the surgical techniques used for the suture of a tendon injury, whether sub-acute or chronic, the surgeon needs to plan in advance the agumentation to use, such as allograft, autotransplantation or reconstruc- tion with synthetic grafts. A surgical access Boyd and Anderson, modified according Morrey ${ }^{16}$ allows a full exposition of the tissue, which is useful to break free from adhesions and inspect any tendon retractions. In these cases, the release of the bicep aponeurosis or the fibrous tendon could help mobilize the distal tendon ${ }^{17}$. Regardless of the surgical technique employed, the biceps tendon is reinserted into the radial tuberosity, which is appropriately worked to accommodate the tendon. The minimally invasive endoscopic has a peculiar indication in type "1" injuries where there is a partial tendon injury. This method involves the arthroscopic portal access on the volar slope to the radial tuberosity in supination and extended elbow. It is used with tourniquet fascia (Fig. 8) to make a bloodless field, it is useful in the dynamic evaluation of the lesion and in the inset exploration tuberosity.

Patient management in the post operative is always performed by the application of a guardian or pinstripe splint for about two weeks at $90^{\circ}$, in order to facilitate the initial reparative processes and reabsorption of the edema, although from the outset patients are granted cautious and gradual movements. Subsequently, they continue assisted active joint mobilization exercises and neuromuscular taping ${ }^{18}$. Muscle strengthening exercises do not begin until at least 2 months after surgery and heavy activity after about 6 months. It is known that surgery is not without complications, which are mainly two types: ectopic that may form between the radius and ulna proximally with pain and reduced joint mobility, and load nerve deficits in the lateral cutaneous nerve, the posterior interosseous nerve or radial nerve ${ }^{19}$. Some Authors have described a different rehabilitation protocol allowing early mobilization of the elbow. This protocol allows mobilization from 4 to 6 weeks after surgical treatment by allowing the active extension of the elbow. Alternatively, patients are allowed to actively ex-

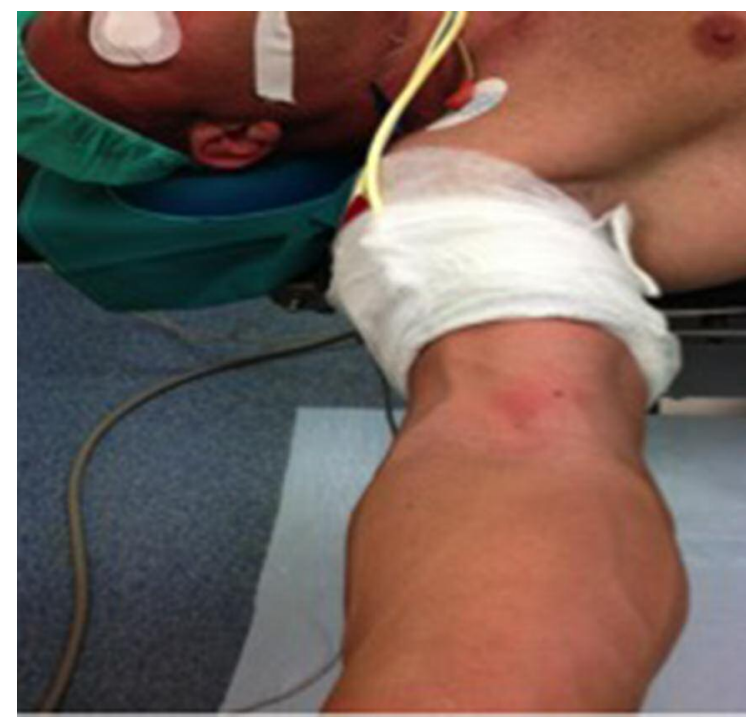

Figure 8. Tourniquet fascia. 
tend the elbow while bending must be passive. Subsequently, the patient is encouraged to actively flex and extend the elbow in addition to active forearm rotation. The results of early surgical treatment for such injuries are very positive, with a satisfactory recovery and a return to sports at the same level before the break, in almost all patients. A special mention should be given to the delayed surgical management of these lesions. In some cases, the diagnosis of bicep tendon rupture was late or the decision to operate was postponed for other reasons. The ideal time to undertake surgical repair is within 2 weeks after injury, during which the bicep tendon can be easily recovered and mobilized. Although, there have been successful cases of tendon recovery up to 3 months after injury. The presence of plentiful degeneration and tendon retraction may make it impossible to mobilize the tendon enough to allow direct repair to the radial tuberosity. Tendon grafts, sometimes reinforced with a ligament augmentation device, have been successfully used ${ }^{8}$. Another alternative is to connect the tendon of the bicep brachii muscle flap to improve the resistance in the elbow flexion, but with no effect on the resistance in supination. Distal lesions of the brachial biceps are infrequent and history is very important for the correct diagnosis. This work was aimed to investigate possible direct and indirect causes that allow us to direct our diagnostic question such as: trauma, metabolic diseases, cigarette smoking, intake of anabolic steroids. In addition, focusing the traumatic mechanism allows us to understand the phases of the injury time. The defining symptom is the painful feeling "pop" at the time of the trauma, described as a feeling of a sudden tear in the anterior region of the elbow followed by a dull ache.

In addition, patients show swelling and a large hematoma in the region of the tendon detachment. A complete rupture occurs with a retraction of the muscle belly and an altered profile of the bicep muscle. Conventional imaging techniques including X-ray, $\mathrm{MRI}$, ultrasound are important to perform preoperatively for a complete classification of the lesion that must be treated ${ }^{20}$. A still underdeveloped method is endoscopy of the distal bicep, which allows us to clearly classify the lesion according to the mechanism of the injury. If the documented lesion is complete, immediate surgical repair is required if you want to maximize the clinical and functional results: the same cannot be said for the partial injury21-24. Among the many widely used techniques for treatment of distal lesions of the brachial bicep tendon available to the transosseous suture are the use of out-type systems in Endobutton ${ }^{\circledR}$ and Zip-Loop ${ }^{\circledR}$ and anchors systems.

\section{Conclusions}

Grewal et al. recently presented the results of a randomized clinical trial evaluating two different surgical approaches for the repair of distal biceps tendon ruptures $^{25}$. However, the best approach for repair (single $v s$ double incision) is still subject of debate ${ }^{26}$. In our study, we examined the treated lesions with two different surgical techniques: single access and dual access. The clinical and functional outcomes during the follow-up examination performed 6-12-24 months after surgery showed excellent results in patients treated with both types of surgical procedures. Only one patient developed acute complications, in fact the patient reported a stupor sensorimotor of the interosseous nerve that persisted for 10 months. This patient was treated with dual access technique (group 2) even if this data is not statistically significant we can assert that the best surgically treatment, in our opinion, is the single incision but further studies are needed to confirm and strengthen our hypothesis.

\section{Acknowledgements}

This study was supported by a Grant-in-Aid provided by FIR 2016, (cod. 314509), University of Catania, Italy. The Authors would like to thank Prof. Iain Halliday for commenting and making corrections to the paper.

\section{Conflict of interest}

The Authors declare that they do not have any conflict of interest.

\section{References}

1. Gabel GT. Acute and chronic tendinopathies at the elbow. Current opinion in rheumatology. 1999;11:138-143.

2. Padulo J, Oliva F, Frizziero A, Maffulli N. Basic principles and recommendations in clinical and field science research. Muscles Ligaments Tendons J. 2014;3:250-252.

3. Oliva F, Misiti F, Maffulli N. Metabolic diseases and tendinopathies: the missing link. Muscles, Ligaments and Tendons $\mathrm{J}$. 2014;4:273-274.

4. Dunkman AA, Buckley MR, Mienaltowski MJ, et al. The injury response of aged tendons in the absence of biglycan and decorin. Matrix Biol. 2014:35:232-238.

5. Khaliq Y, Zhanel GG. Fluoroquinolone-associated tendinopathy: a critical review of the literature. Clin Infect Dis. 2003;36: 1404-1410.

6. Järvinen TA, Kannus P, Maffulli N, Khan KM. Achilles tendon disorders: etiology and epidemiology. Foot Ankle Clin. 2005; 10:255-266.

7. Morrey BF, Sanchez-Sotelo J. The elbow and its disorders. Elsevier Health Sciences, 2009.

8. Morrey BF. Tendon injuries and tendinopathies about the elbow. Orthopaedic knowledge update. Shoulder and elbow. American Academy of Orthopaedic Surgeons Rosemont (IL). 1997;337-344.

9. Eames MH, Bain GI, Fogg QA, van Riet RP. Distal biceps tendon anatomy: a cadaveric study. J Bone Joint Surg Am. 2007;89:1044-1049.

10. Mazzocca AD, Burton KJ, Romeo AA, Santangelo S, Adams DA, Arciero RA. Biomechanical evaluation of 4 techniques of distal biceps brachii tendon repair. Am J Sports Med. 2007; 35:252-258. 
11. Padulo J, Oliva F, Frizziero A, Maffulli N. Muscles, Ligaments and Tendons Journal - Basic principles and recommendations in clinical and field Science Research: 2016 Update. MLTJ. 2016;6(1):1-5.

12. Kelly EW, O'Driscoll SW. Mini-incision technique for acute distal biceps tendon repair. Techniques in Shoulder \& Elbow Surgery. 2002;3:1:57-62.

13. Castrogiovanni P, Trovato FM, Szychlinska MA, et al. Effects of Synthetic Anti-Inflammatory Sterol in CB3V-Induced Myocarditis: A Morphological Study on Heart Muscle Tissue. J Funct Morphol Kinesiol. 2016;1:69-89.

14. Boyd HB, Anderson LD. A method for reinsertion of the distal biceps brachii tendon. J Bone Joint Surg Am. 1961;43.7:10411043.

15. Morrey BF. Tendon injuries about the elbow. The elbow and its disorders. 1993:2:492-504.

16. Morrey BF. Distal biceps tendon rupture. The elbow. 1994; 115-128.

17. Noble JS. Chronic distal biceps tendon ruptures: evaluation, treatment options and management using an autogenous semitendinosus technique. Techniques in Shoulder \& Elbow Surgery. 2003;4.4:145-153.

18. Castrogiovanni P, Di Giunta A, Guglielmino C, et al. The Effects of Exercise and Kinesio Tape on Physical Limitations in Patients with Knee Osteoarthritis. J Funct Morphol Kinesiol. 2016;1:355-368.
19. Pavone V, Riccioli M, Testa G, et al. Surgical Treatment of Displaced Supracondylar Pediatric Humerus Fractures: Comparison of Two Pinning Techniques. J Funct Morphol Kinesiol. 2016;1:39-47.

20. Cannavò L, Costarella L, Pavone V, Testa G, De Gori M, Sessa G. Arthrodesis and Hemiarthroplasty: Different Techniques in the Treatment of Hallux Rigidus-Surgery and Postoperative Rehabilitation. J Funct Morphol Kinesiol. 2016;1:102-108.

21. Dürr HR, Stäbler A, Pfahler M, Matzko M, Refior HJ. Partial rupture of the distal biceps tendon. Clin Orthop Relat Res. 2000;374:195-200

22. Bernstein AD, Breslow MJ, Jazrawi LM. Distal biceps tendon ruptures: a historical perspective and current concepts. American journal of orthopedics (Belle Mead, NJ). 2001;30.3:193200.

23. Bain GI, Johnson LJ, Turner PC. Treatment of partial distal biceps tendon tears. Sports Med Arthrosc. 2008;16:154-161.

24. Eames MHA, Bain Gl. Distal biceps tendon endoscopy and anterior elbow arthroscopy portal. Techniques in Shoulder \& Elbow Surgery. 2006;7.3:139-142.

25. Grewal R, Athwal GS, MacDermid JC, et al. Single versus double-incision technique for the repair of acute distal biceps tendon ruptures: a randomized clinical trial. J Bone Joint Surg Am. 2012;94:1166-1174.

26. Kodde IF, van den Bekerom MP, Eygendaal D. Best approach for the repair of distal biceps tendon ruptures. World J Orthop. 2013;4:98-99. 